\title{
Role of adsorbates on dynamics of hot-electron (type I and II) thermalization within gold nanoparticles
}

\author{
Christophe Bauer *, Jean-Pierre Abid, Hubert H. Girault \\ Laboratoire d'électrochimie physique et analytique, faculté des sciences de base, École polytechnique fédérale de Lausanne, \\ 1015 Lausanne, Switzerland
}

Received 30 August 2004; accepted after revision 18 March 2005

Available online 15 September 2005

\begin{abstract}
Early stages of hot-electron thermalization in small gold nanoparticles wrapped in an adsorbates shell have been investigated by femtosecond transient absorption spectroscopy. Type-I hot electrons thermalize in $800 \mathrm{fs}$ (to form type-II hot electron) either by scattering with cold conduction band electrons or by chemical interface scattering with adsorbates shell. Type-II hot electrons redistribute the excess energy toward the lattice via electron-phonon coupling in 1.8-3.6 ps depending on pump fluence. The electron-phonon coupling process (type II hot electron) is retarded because of the incomplete internal thermalization of type-I hot electron at early times due to the presence of adsorbates. To cite this article: $C$. Bauer et al., C. R. Chimie 9 (2006). (c) 2005 Académie des sciences. Published by Elsevier SAS. All rights reserved.
\end{abstract}

\section{Résumé}

Les premiers événements relatifs à la thermalisation des électrons chauds dans des nanoparticules d'or enrobées dans une coquille d'adsorbats ont été étudiés par spectroscopie femtoseconde d'absorption transitoire. Les électrons chauds de type I se thermalisent en 1 ps (pour former les électrons chauds de type II), soit par diffusion avec les électrons froids de conduction, soit par diffusion interfaciale chimique dans la coquille d'adsorbats. Les électrons chauds de type II redistribuent l'énergie en excès en 1,6 à 3,6 ps en fonction de la puissance de pompe. Le processus de couplage électrons-phonon est retardé dans ce système en raison de la dépopulation incomplète des électrons de type I induite par la présence d'adsorbats durant les premiers temps. Pour citer cet article : C. Bauer et al., C. R. Chimie 9 (2006).

(C) 2005 Académie des sciences. Published by Elsevier SAS. All rights reserved.

Keywords: Metal nanoparticles; Gold; Ultrafast spectroscopy; Hot electrons; Adsorbates; Electron tunneling

Mots-clés : Nanoparticules métalliques ; Or ; Spectroscopie ultrarapide ; Électrons chauds ; Adsorbats ; Effet tunnel

\footnotetext{
* Corresponding author.

E-mail address: christophe.bauer@epfl.ch (C. Bauer).
} 


\section{Introduction}

The understanding of hot electron dynamics in metals and semiconductors and their interactions with adsorbates is of fundamental importance due to the high potential of nonequilibrium charge carriers for enhancing the photon conversion efficiency $[1,2]$. The investigation of the interaction of femtosecond laser pulses with metal nanoparticles (NPs) is a growing field due to the unique possibility of creating a far from equilibrium situation in low-dimensional media [3-5]. Electron tunneling at metal surfaces underlies many redox processes and is at the heart of electrochemistry. Small NPs are excellent candidates to investigate electronic surface phenomena because of their very high surfaceto-volume ratios. Despite of intensive investigations of electron-phonon coupling processes in metal nanoparticles, a clear picture of the fundamental parameters that govern hot electron relaxation dynamics is still not completely available. For example, size effects on electronphonon coupling rates are still under discussion for gold nanoparticles below $10 \mathrm{~nm}$ in diameter [6]. The perturbation of the electron distribution in a metal due to the absorption of a femtosecond laser pulse leads to the creation of nascent nonthermal electrons (NNEs). The classical picture for the different events of NNEs relaxation occurring in metal following an external optical energy input involves three main processes [1]: $(i)$ electron-electron scattering $\left(\mathrm{P}_{\mathrm{I}}\right),($ ii $)$ electron-phonon interaction $\left(\mathrm{P}_{\mathrm{II}}\right)$ and (iii) phonon-medium interaction $\left(\mathrm{P}_{\mathrm{III}}\right)$. In bulk metal, NNEs redistribute the energy by e-e scattering with free 'cold' conduction band electrons giving rise to a well defined temperature for the hot electron gas (internal thermalization process $\mathrm{P}_{\mathrm{I}}$, decay of type-I hot electron). The electronic temperature rise varies from hundreds to thousands of Kelvin depending on the laser pump fluence. The hot electron gas transfers the energy into the lattice by electron-phonon interaction until the electron gas and the lattice are in thermal equilibrium (external thermalization process $\mathrm{P}_{\mathrm{II}}$, decay of type-II hot-electron). In the last step, the phonons transfer their energy to the surrounding medium (heat dissipation process $\mathrm{P}_{\mathrm{III}}$ ) within hundreds of picoseconds. This description includes the assumptions of the two-temperature model, which is the most widely used model to describe the interaction of a femtosecond laser pulse with a metal [7]. These different stages of energy dissipation are not necessary sequential but can overlap in time.
In this paper, we report on thermalization dynamics of type-I and -II hot electrons by femtosecond transient absorption pump-probe spectroscopy of gold NPs with a diameter of $4.2 \mathrm{~nm}$ wrapped in a shell of sulfate. The purpose is to obtain information on the effect of adsorbates on hot electron thermalization. Concerning the vocabulary used in this paper, NNEs are type-I hot electrons that are neither in equilibrium with others conduction electrons nor with the lattice, whereas type-II hot electrons are in equilibrium with other electrons but not yet with the lattice. Finally, thermalized electrons are those in thermal equilibrium with the lattice. Here, we show that the presence of adsorbates provides an additional decay channel for NNEs. This additional mechanism has been identified as the ultrafast chemical interface scattering (UCIS) [8]. The electron tunneling back and forth between the adsorbates and the metal induces retardation of type-I hot electron decay because of the creation of a quasi-stationary nonequilibrium state during $\sim 1$ ps. Furthermore, we observed that UCIS process can also slow down electronphonon coupling process (decay of type-II hot electrons). Thus, retardation of hot-electron relaxation by adsorbates can be due to the slow down of electronelectron scattering in the present system.

\section{Experimental methods}

\subsection{Samples preparation}

The three dimensions $\mathrm{Au} / \mathrm{TiO}_{2}$ nanoarchitectures composite were prepared by wet-grafting of initial $\mathrm{Au}$ NPs of $1.7 \mathrm{~nm}$ in diameter derivatized with mercapto succinic acid (MSA) to a $\mathrm{TiO}_{2}$ mesoporous thin film. The elementary $\mathrm{TiO}_{2}$ particle size is $15 \mathrm{~nm}$ and the thickness of the mesoporous films measured by profilometry is $5 \mu \mathrm{m}$. Before the insertion of gold NPs, the $\mathrm{TiO}_{2}$ nanocrystalline films exhibit a porosity of $60 \%$ with an average size of $20 \mathrm{~nm}$ for the pores. Aqueous solution of $1.7 \mathrm{~nm}$ gold NPs were synthesized by $\mathrm{NaBH}_{4}$ reduction of $\mathrm{AuCl}_{4}{ }^{-}$in a mixture of water and methanol. The synthesis including five consecutive washing and centrifugation steps with pure methanol. This sequence ensures the removal of inorganic ions. The solvent excess was evaporated at temperatures below $313 \mathrm{~K}$ and pressure under $5 \times 10^{-3}$ torr for $12 \mathrm{~h}$. The core size of the resulting particles exhibited an aver- 
age diameter of $(1.7 \pm 0.4) \mathrm{nm}$. The final gold NPs used to perform laser spectroscopy measurements was obtained by heat treatment at $723 \mathrm{~K}$ for $30 \mathrm{~min}$ generating gold NPs of $(4.2 \pm 0.5) \mathrm{nm}$ in diameter. The size of gold NPs has been revealed by transmission electronic microscopy using a Philips CM20.

\subsection{Femtosecond spectroscopy}

The femtosecond transient absorption set-up features a compact Ti:Sa-amplified femtosecond laser with a repetition rate of $1 \mathrm{kHz}$, a fundamental wavelength at $775 \mathrm{~nm}$, a pulse width of $120 \mathrm{fs}$ and an energy of $1 \mathrm{~mJ}$ per pulse. The output beam was split into three parts for pumping two double-stage noncollinear optical parametric amplifiers (NOPA) and to produce a white light continuum in a 2-mm-thick sapphire plate. The output pulses of the NOPAs were compressed in SF10glass prism pair compressors to obtain $50 \mathrm{fs}$ pulses. Pump and probe beams were directed toward a parabolic mirror that focused them into the sample. Silicon diode was placed directly after the samples to measure the transmitted light intensity for the monochromatic probing, whereas for transient spectra acquisition a broadband membrane beam splitter was placed before the sample to split the white light beam into signal and reference arms. Both were sent into a Triax 320 spectrograph. Reference and signal beams were individually detected by diode array detectors. The signal from the detector was connected to a SR-830 lock-in amplifier tuned at $220 \mathrm{~Hz}$ by a chopper modulating the pump beam. The measurements were performed at room temperature and within the low-perturbation regime, the electronic temperature rise $\left(\Delta T_{\mathrm{e}}\right)$ was estimated to be in the range of 50-300 K depending on pump fluences.

\section{Results and discussion}

Fig. 1A shows UV/Vis spectra of gold NPs wrapped in an adsorbates shell and calculations taking into account confinement [8,9] according to Mie's theory [10]. The measured absorption spectrum (a) contains absorption arising mainly from the band gap of $\mathrm{TiO}_{2}$ $(3.1 \mathrm{eV})$ and the electronic interband transitions in gold NPs $(2.3 \mathrm{eV}$ ) below 390 and $540 \mathrm{~nm}$, respectively. The band with an absorption maximum at $526 \mathrm{~nm}(2.36 \mathrm{eV})$ is called the surface plasmon band and corresponds to
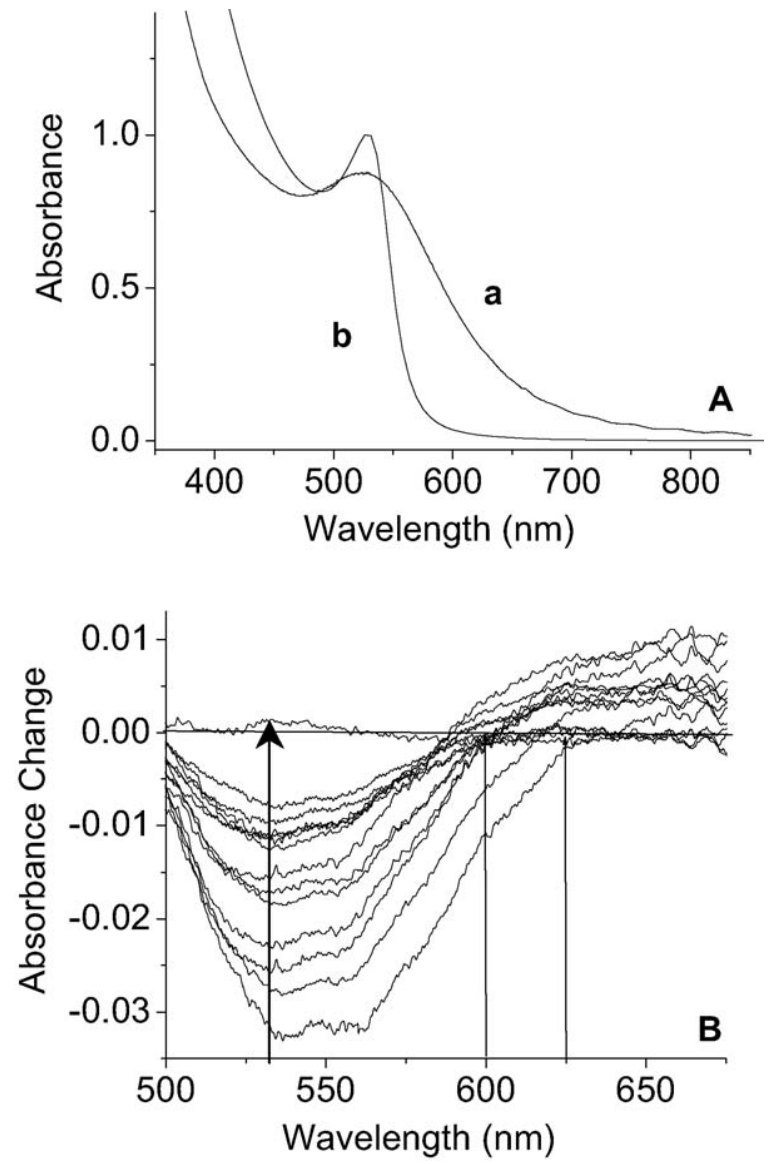

Fig. 1. A. (a) Experimental and (b) Calculated (according to Mie's theory) UV/Visible absorption spectra of the gold NPs embedded in $\mathrm{TiO}_{2}$ matrix. B. Femtosecond transient absorption spectra after pumping at $480 \mathrm{~nm}$ using the broadband light continuum recorded with a delay time step of $500 \mathrm{fs}$ between the pump and the probe pulse.

the collective oscillations of conduction electrons across NPs. The experimental spectrum (a) for these 4.2-nm gold NPs deviates strongly from the calculated spectrum (b) according to Mie's theory [8]. The large nonclassical broadening of the surface plasmon band is attributed to the so-called chemical interface damping [9-12]. This observation indicates that electrons can tunnel back and forth between metal nanoparticles and empty electronic states of adsorbates. Fig. 1B shows differential transient absorption spectra upon pumping at $480 \mathrm{~nm}$ using the broadband light continuum recorded with a delay time step of $500 \mathrm{fs}$ between each spectra from -250 fs to $6.25 \mathrm{ps}$. Spectra at early stages have been identified to be linked to NNEs, whereas spectra after 4 ps are attributed to the hot electron gas 
[8]. NNEs exhibit broad bleach with negligible absorption. On the opposite, the internally hot thermalized electron gas shows a narrow bleach with a positive absorption for wavelengths longer than $590 \mathrm{~nm}$. The observed differential transient absorption spectra are in excellent accordance with calculations reported in the literature $[13,14]$. Upon comparison of the spectra recorded at $250 \mathrm{fs}$ and times longer than $3 \mathrm{ps}$, a time dependent shift of the crosspoint of the signal with the baseline from 630 to $585 \mathrm{~nm}$ is clearly observed between these spectra.

To extract time constants for the internal thermalization process (e-e scattering, type-I hot electron), we use two-color pump-probe spectroscopy to monitor (i) the decay of NNEs and (ii) the formation of type II hot electron gas (build-up of Fermi-Dirac distribution). Transient absorption data obtained at $625 \mathrm{~nm}$ are shown in Fig. 2A. The signal can be well fitted with the following expression:

$S(t)=A \exp \left[1-\exp \left(-t / \tau_{1}\right)\right] \exp \left[-t / \tau_{2}\right]+B$

where the delayed rise time is introduced by the first term $\left(\tau_{1}\right)$, which describes the formation of type-I hot electron, whereas $\tau_{2}$ corresponds to the energy exchange between the hot-electron gas and the lattice (external thermalization) and the constant B accounts finally for the slow decay linked to the heat dissipation from gold NPs into the matrix. After deconvolution of the pumpprobe cross correlation, the solid line obtained with Eq. (1) reproduces well the experimental data (open circles) giving $\tau_{1}=540 \mathrm{fs}$ and $\tau_{2}=3.6 \mathrm{ps}$. The internal thermalization time $\left(\tau_{1}\right)$ can also be obtained by interrogating the temporal shift and the narrowing of the transient spectra with the probe wavelength at $600 \mathrm{~nm}$ as illustrated in Fig. 2B. During the bleach recovery, the signal is modulated due to the superposition of oscillations. The mechanism of generation of these oscillations is beyond the scope of this paper and will be discussed in detail in a separate study. The experimental data shown in Fig. 2B can be very well fitted with the following expression:

$S(t)=C \exp \left[-t / \tau_{1}\right]+D \cos [2 \pi v t+\varphi] \exp \left[-t / \tau_{3}\right]+E$

where $\tau_{1}$ is the bleach decay time constant which reflects the internal thermalization, $v$ is the frequency of the oscillations, $\varphi$ is the phase and $\tau_{3}$ the damping time constant, $C$ and $D$ are the amplitudes for the expo-
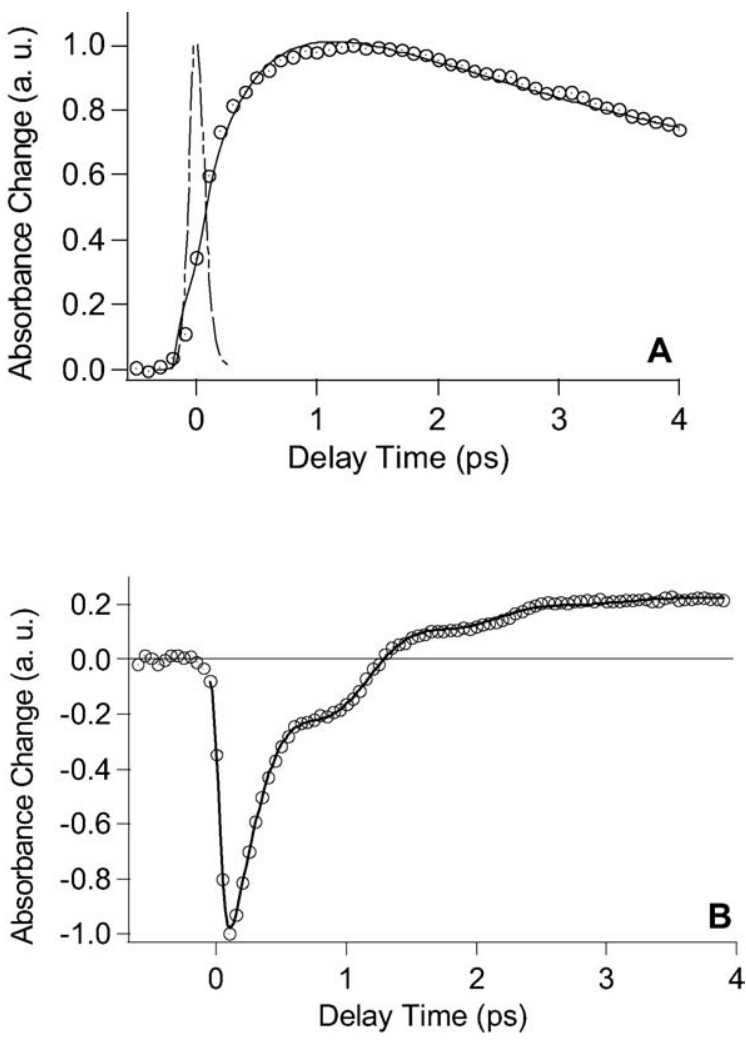

Fig. 2. A. Transient absorption data after pumping at $500 \mathrm{~nm}$ and probing at $625 \mathrm{~nm}$ for pump fluence of $500 \mathrm{~nJ}$ per pulse for gold NPs. The dashed line represents the pump-probe cross correlation of the instrument. B. Transient absorption data after pumping at $525 \mathrm{~nm}$ and probing at $600 \mathrm{~nm}$ for pump intensity at $675 \mathrm{~nJ}$ per pulse. The solid lines in Fig. 2A and B are fits of the experimental data according to equations (1) and (2), respectively.

nential decay and the damped cosinus function, respectively and the constant $E$ stands for the offset. The excellent fit after convolution of the pump-probe cross correlation of the experimental data confirm the existence of a single recovery process resulting in the following time constants: $\tau_{1}=(767 \pm 11) \mathrm{fs}, v=1.02 \mathrm{THz}$, $\tau_{3}=(720 \pm 55)$ fs. Therefore, the bleach recovery recorded at $600 \mathrm{~nm}$ is a direct signature of the depopulation of nonthermal electrons during the build-up of the Fermi-Dirac distribution in accordance with transient absorption spectra. The difference for $\tau_{1}$ obtained in probing at 600 and $625 \mathrm{~nm}$ is due to the fact that closer to Fermi energy, longer internal thermalization times are expected according to Fermi liquid theory.

Time constants $\left(\tau_{1}\right)$ of e-e scattering (decay of NNEs, type-I hot electrons) observed here are more than four times longer than for gold NPs with similar sizes 
[14]. In accordance with the information from the nonclassical broadening of the surface plasmon, we attribute the retardation of the internal thermalization to the presence of an additional decay channel for NNEs in these small gold nanoparticles. NNEs experience an inelastic resonant scattering process into unoccupied molecular orbitals of adsorbates, as schematically illustrated in Fig. 3. Within this process, the photonic energy is not redistributed among other cold conduction band electrons, but is directly transferred toward the adsorbates via the excitation of vibrational modes. It should be noted that the excitation could be directly localized in the adsorbates [15]. Depending on the amount of energy deposited by NNEs, adsorbates can undergo surface chemistry. The two-temperature model (TTM) is no longer valid for this system because of $(i)$ the temporal overlap between the internal and external thermalization process is very important (ii) a part of the

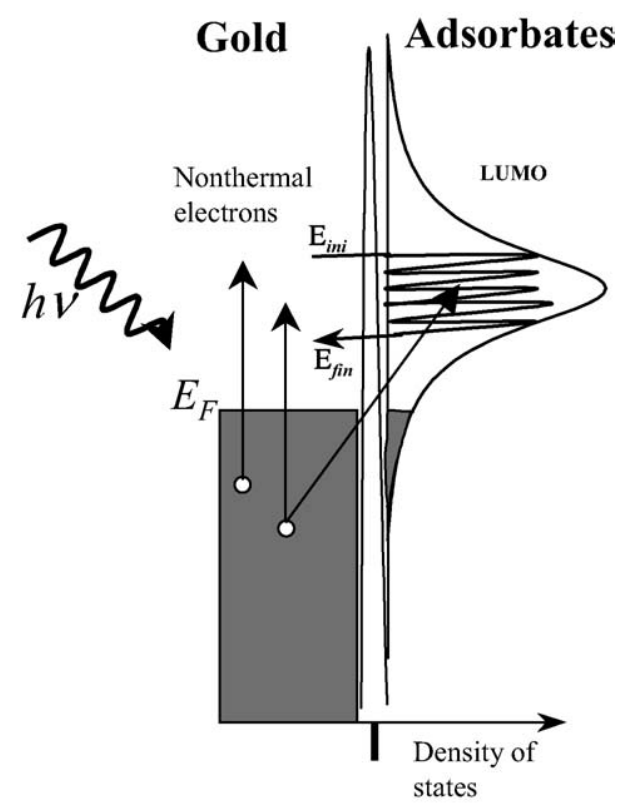

Fig. 3. Schematic drawing of the Ultrafast Chemical Interface Scattering (UCIS) process in metal NPs. Immediately after absorption of the femtosecond pulse, a nonequilibrium distribution of nascent nonthermal electrons (as photoexcited) is created with energies $E_{\text {ini }}$ in the range for intraband transition $E_{\mathrm{F}} \leq E_{\mathrm{ini}}<E_{\mathrm{F}}+h v$. The electron is temporary transferred into an empty electronic level with an energy $E_{\text {ini }}$ with the subsequent creation of an intermediate negative ion state. The electron then scatters back with energy $E_{\text {fin }}$ into the gold metal conduction band. The nascent nonthermal electrons have deposited energy $\Delta E=E_{\mathrm{ini}}-E_{\mathrm{fin}}$ in the affinity electronic levels of adsorbates and is converted in molecular vibrational energy. It should be noted that the excitation can be directly localized in the adsorbates. photonic energy is directly transferred toward the adsorbates (not among 'cold' conduction band electrons). These findings show that adsorbates can scavenge type-I hot electrons via an inelastic tunneling process.

To investigate the decay of type-II hot electrons, we tune the probe wavelength close to the maximum of the plasmon band $(530 \mathrm{~nm})$ since this spectral region is very sensitive to the electron-phonon coupling process (decay of type-II hot electrons) [8]. Fig. 4A shows femtosecond data obtained with a probe wavelength at $530 \mathrm{~nm}$ for different pump fluences upon pumping at $480 \mathrm{~nm}$. The main feature is the dependence of the recovery rate on the initial electronic temperature rise. Time constants for electron-phonon coupling vary from
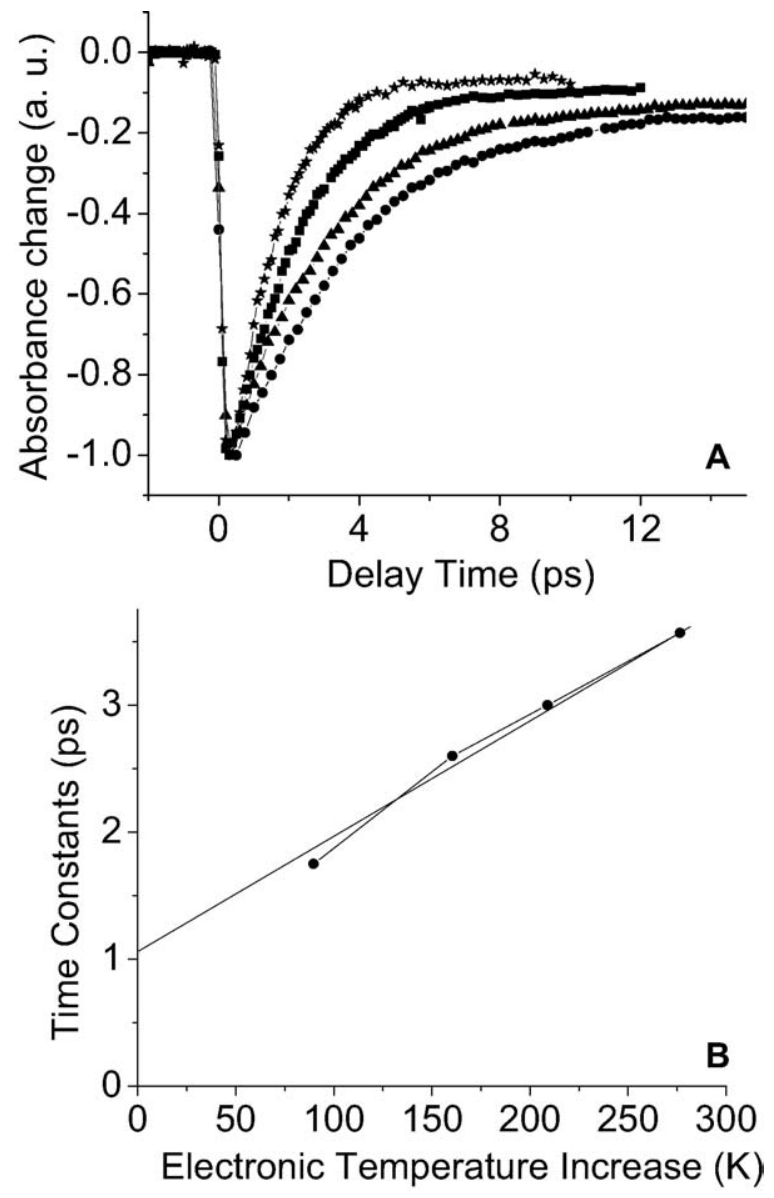

Fig. 4. A. Transient absorption data after pumping at $480 \mathrm{~nm}$ and probing at $530 \mathrm{~nm}$ for gold NPs with an electronic temperature increase of 90, 160, 210 and $280 \mathrm{~K}$ for stars, squares, triangle and circles, respectively. B. Time constants extracted from a singleexponential decay of type-II hot electrons as a function of the initial electronic temperature rise. 
1.8 to 3.6 ps. This effect has been observed earlier and can be rationalized by the fact that the electronic heat capacity is also dependent on the initial electronic temperature rise $[16,17]$. Fig. 4B shows time constants of the decay of type-II hot electron as a function of the calculated electronic temperatures of the hot electron gas ${ }^{1}$. Time constants of electron-phonon coupling decrease almost linearly with the pump fluence. Due to pump fluence dependences and deviations from an exponential decay (due to incomplete e-e scattering at early times), it is rather difficult to extract an absolute electron-phonon coupling time constants. However, an extrapolation to zero pump fluence can be an approach for comparison with other systems [6,17]. The intercept of the slope in Fig. 4B, leads to a zero power limit of $1.1 \mathrm{ps}$ for the present gold/adsorbates system. The value obtained for this metal/adsorbates nanosystem is much higher than those reported for other gold NPs systems [18], which is around 650 fs. Thus, we observe for the present system a slow down of electron-phonon coupling rates compared to 'naked' gold NPs. Relatively long electron-phonon coupling time constants have been observed very recently for metal nanoparticles or nanorods coated with adsorbates [19-21]. Our study suggests that the slow hot electron thermalization in the presence of adsorbates is due to the slow internal thermalization of type-I hot electrons. Indeed, as previously reported, the rate of electron-phonon coupling increases as long as the internal thermalization (decay of type-I hot electron) is in progress $[22,23]$. In the nonthermal regime, the electron-phonon coupling rate is in fact not constant but evolves with time and the change of nonthermal electron states. Finally, the scenario that comes from femtosecond data for dynamical events can be described as follows: The attachment of type-I hot electron to adsorbates decreases the internal thermalization rate (e-e scattering) because of the creation of a quasistationary nonequilibrium state, this latter process also affects the decay of type-II hot electron. This dynamical

\footnotetext{
${ }^{1}$ The electronic temperature rise was estimated from the gold heat capacity, the adsorbed optical energy (according to the laser pulse intensity, the spot size and the sample absorbance) and the concentration of gold nanoparticles. However, these values should be taken with care since there are uncertainties on the spot size. Furthermore, as demonstrated in this work, a significant part of the optical energy is directly dissipated toward adsorbates and is therefore not redistributed among other electrons to build-up the Fermi-Dirac distribution.
}

picture provides a possible explanation for the slow down of electron-phonon coupling rate observed for gold nanoparticles coated with adsorbates [19-21]. We have performed measurements with very-low pump power (less than one photon per nanoparticle in average) that confirm the results from zero power extrapolation presented here.

\section{Conclusion}

The role of adsorbates on the relaxation of type-I and -II hot electrons has been investigated by femtosecond transient absorption spectroscopy in a gold/sulfate nanosystem. Analysis of the surface plasmon broadening in combination with ultrafast studies of the internal thermalization allowed to identify, in addition to the classical e-e scattering mechanism, an alternative decay channel for nascent nonthermal electrons in small gold NPs, i.e. the ultrafast chemical interface scattering (UCIS) mechanism. Nascent nonthermal electrons experience an inelastic resonant scattering process into unoccupied molecular orbitals of adsorbates. Our results show that adsorbates can directly quench type I hot electrons in metal nanoparticles. Energy gained during UCIS process by adsorbates, via the excitation of molecular vibrational modes, can be used to drive surface chemistry. Therefore, within this scenario, the excess photonic energy is not lost as heat but can be harnessed by the adsorbates to undergo chemistry.

Furthermore, adsorbates-induced retardation of internal thermalization due to the appearance of a quasistationary nonequilibrium state was observed, the decay of type-I hot electron occurs in $\sim 1$ ps. electron-phonon coupling time constant measured for the present system (1.1 ps) is higher compared to 'naked' gold NPs. The incomplete thermalization of type-I hot electrons slows down the decay of type-II hot electrons due to the longlived nonthermal regime. This observation provides insight as to why adsorbates-affected hot electron thermalization [19-21]. Indeed, the slow decay of type-II hot electron is attributed to a long-lived nonthermal regime.

\section{Acknowledgments}

The authors gratefully acknowledge Pascal Comte and Gerrit Boschloo for the preparation of the titanium 
dioxide colloids, Valérie Devaud for the technical assistance. The present work is financed by the 'Fonds national suisse de la recherche scientifique' and the 'Office fédérale de l'éducation et de la science' (project C02.0010). The 'Laboratoire d'électrochimie physique et analytique' is part of the TMR network SUSANA (Supramolecular Self-Assembly of Interfacial Nanostructures).

\section{References}

[1] R.J.D. Miller, G.L. McLendon, A.J. Nozik, W. Schmickler, F. Willig, Surface Electron-Transfer Processes, Wiley-VCH, New York, 1995.

[2] A.J. Nozik, Annu. Rev. Phys. Chem. 52 (2001) 193.

[3] S. Link, M.A. El-Sayed, J. Phys. Chem. B 103 (1999) 8410.

[4] J.H. Hodak, A. Henglein, G.V. Hartland, J. Phys. Chem. B 104 (2000) 9954.

[5] C. Voisin, N.D. Del Fatti, D. Christofilos, F. Vallée, Phys. Rev. B 105 (2001) 2264.

[6] G.V. Hartland, Int. J. Nanotechnol. 1 (2004) 307.

[7] S.I. Animisov, B.L. Kapeliovich, T.L. Perel'man, Sov. Phys. JETP 39 (1974) 375.

[8] C. Bauer, J.-P. Abid, D. Fermin, H.H. Girault, J. Chem. Phys. 120 (2004) 9302.
[9] U. Kreibig, M. Vollmer, Optical Properties of Metal Clusters, Springer, Berlin, 1995.

[10] G. Mie, Ann. Phys. 25 (1908) 377.

[11] B.N.J. Persson, Surf. Sci. 283 (1993) 153.

[12] H. Hövel, S. Fritz, A. Hilger, U. Kreibig, Phys. Rev. B 48 (1993) 18178.

[13] N. Del Fatti, F. Vallée, Appl. Phys. B 73 (2001) 383.

[14] C. Voisin, D. Christofilos, P.A. Loukakos, N.D. Del Fatti, F. Vallée, J. Lermé, M. Gaudry, E. Cottancin, M. Pellarin, M. Broyer, Phys. Rev. B 69 (2004) 195416.

[15] H. Petek, H. Nagano, M.J. Weida, S. Ogawa, J. Phys. Chem. B 105 (2001) 6767.

[16] J.H. Hodak, I. Martini, G.V. Hartland, Chem. Phys. Lett. 284 (1998) 135.

[17] J.H. Hodak, A. Henglein, G.V. Hartland, J. Chem. Phys. 112 (2000) 5942.

[18] S. Link, C. Burda, Z.L. Wang, M.A. El-Sayed, J. Chem. Phys. 111 (1999) 1255.

[19] S.L. Westcott, R.D. Averitt, J.A. Wolfgang, P. Nordlander, N.J. Halas, J. Phys. Chem. B 105 (2001) 9913.

[20] S. Link, A. Furube, M.B. Mohamed, T. Asahi, M.H. El-Sayed, M.A. El-Sayed, J. Phys. Chem. B 106 (2002) 945.

[21] S. Link, D.J. Hathcock, B. Nikoobakht, M.A. El-Sayed, Adv. Mater. 15 (2003) 393.

[22] R.H.M. Groeneveld, R. Sprik, A. Lagendijk, Phys. Rev. B 51 (1995) 11433.

[23] N. Del Fatti, R. Bouffanais, F. Vallée, C. Flytzanis, Phys. Rev. Lett. 81 (1998) 922. 